ARTICLE

DOI: $10.1038 / s 41467-018-07346-z$

\title{
Daily changes in phytoplankton lipidomes reveal mechanisms of energy storage in the open ocean
}

\author{
Kevin W. Becker (10 1, James R. Collins (10) 1,2,6, Bryndan P. Durham³, Ryan D. Groussman², \\ Angelicque E. White (iD ${ }^{4}$, Helen F. Fredricks ${ }^{1}$, Justin E. Ossolinski ${ }^{1}$, Daniel J. Repeta ${ }^{1}$, Paul Carini (iD) ${ }^{5,7}$, \\ E. Virginia Armbrust ${ }^{3} \&$ Benjamin A.S. Van Mooy (D) ${ }^{1}$
}

Sunlight is the dominant control on phytoplankton biosynthetic activity, and darkness deprives them of their primary external energy source. Changes in the biochemical composition of phytoplankton communities over diel light cycles and attendant consequences for carbon and energy flux in environments remain poorly elucidated. Here we use lipidomic data from the North Pacific subtropical gyre to show that biosynthesis of energy-rich triacylglycerols (TAGs) by eukaryotic nanophytoplankton during the day and their subsequent consumption at night drives a large and previously uncharacterized daily carbon cycle. Diel oscillations in TAG concentration comprise $23 \pm 11 \%$ of primary production by eukaryotic nanophytoplankton representing a global flux of about $2.4 \mathrm{Pg} \mathrm{C}_{\mathrm{yr}}{ }^{-1}$. Metatranscriptomic analyses of genes required for TAG biosynthesis indicate that haptophytes and dinoflagellates are active members in TAG production. Estimates suggest that these organisms could contain as much as $40 \%$ more calories at sunset than at sunrise due to TAG production.

\footnotetext{
${ }^{1}$ Department of Marine Chemistry and Geochemistry, Woods Hole Oceanographic Institution, Woods Hole, MA 02543, USA. ${ }^{2}$ Massachusetts Institute of Technology/Woods Hole Oceanographic Institution Joint Program in Oceanography, Woods Hole, MA 02543, USA. ${ }^{3}$ School of Oceanography, University of Washington, Seattle, WA 98195, USA. ${ }^{4}$ College of Earth, Ocean, and Atmospheric Sciences, Oregon State University, Corvallis, OR 97331, USA.

${ }^{5}$ Department of Microbiology, Oregon State University, Corvallis, OR 97331, USA. ${ }^{6}$ Present address: School of Oceanography and eScience Institute, University of Washington, Seattle, WA 98195, USA. ${ }^{7}$ Present address: Department of Soil, Water and Environmental Science, University of Arizona, Tucson, AZ 85721, USA. Correspondence and requests for materials should be addressed to B.A.S.V.M. (email: bvanmooy@whoi.edu)
} 
$\mathrm{D}$ iel cycles in sunlight represent an important source of environmental variability for plankton communities in the oligotrophic subtropical gyres of the world's ocean. This is particularly true for phytoplankton, which depend on sunlight to drive the biosynthesis of macromolecules essential for growth. These organisms possess numerous metabolic capabilities that allow them to survive in darkness, when they are effectively deprived of their primary energy source. For example, diel cycles in phytoplankton gene expression patterns reveal widespread coordination of metabolic pathways to optimize internal biochemical energy allocation ${ }^{1-3}$. However, the attendant diel cycles in the biochemical composition of phytoplankton communities and the biogeochemical consequences of these changes for the ocean carbon cycle remain poorly understood. As subtropical gyres of the oligotrophic ocean are the world's largest biomes, account for about $40 \%$ of the Earth's surface area, and contribute significantly to global productivity and carbon export ${ }^{4,5}$, diel cycles in phytoplankton metabolism are expected to be engrained in the roles these environments play in the global carbon cycle.

A common strategy for energy allocation in phytoplankton centers on the daytime accumulation of energy storage molecules that can then be used to drive metabolism and respiratory activity during the night ${ }^{6,7}$. Laboratory cultures of phytoplankton display clear diel cycles in storage lipid accumulation ${ }^{8-10}$, but whether this metabolic behavior is manifested in the ocean is an open question. While cyanobacteria store energy in the form of carbohydrates (mainly glycogen), eukaryotic phytoplankton predominantly accumulate neutral lipids, mainly in the form of triacylglycerols (TAGs). TAGs are more effective energy stores than carbohydrates, because they contain more chemical energy per mole of carbon and larger quantities can be stored inside the cell as they are non-polar and anhydrous (i.e., lacking a hydration shell) ${ }^{11}$. Thus, large phytoplankton, such as diatoms and dinoflagellates, have a greater capacity for energy storage when compared with small cyanobacterial phytoplankton, such as Prochlorococcus and Synechococcus ${ }^{12}$. The accumulation of energy storage molecules in the form of glycogen and/or TAGs is apparently also a strategy for phytoplankton to deal with unfavorable growth conditions ${ }^{13}$. In the oligotrophic surface waters of the subtropical gyres, nitrogen is persistently scarce; this scarcity has been linked in cultures of many eukaryotic phytoplankton to enhanced TAG accumulation ${ }^{13}$. Thus, in subtropical gyres, we hypothesize that TAGs could play a number of roles in eukaryotic phytoplankton metabolism, contribute to the ability of eukaryotic phytoplankton to compete with cyanobacteria, and thereby play a previously uncharacterized role in the carbon cycle of these environments.

Oscillations in phytoplankton storage lipids have the potential to play important roles in biogeochemical carbon cycling and energy flow through the broader microbial community. For example, the biochemical composition of phytoplankton defines their nutritional quality ${ }^{14,15}$, which has implications for the population dynamics of the zooplankton that graze on them. Yet, field studies on diel periodicity of phytoplankton biochemical composition are rare ${ }^{16-18}$. To this end, we investigated the lipid composition of the planktonic community (i.e., the metalipidome) at high temporal resolution over multiple day/night cycles during two research cruises to the North Pacific Subtropical Gyre (NPSG). Complementary analyses of the plankton community metatranscriptome were conducted to help constrain the sources of the lipids we observed. Comparison to measurements of primary productivity showed that diel synthesis and consumption of TAGs account for a large fraction of the flux of carbon and energy through the ecosystem.

\section{Results}

Diel patterns of phytoplankton lipids. During a cruise in the NPSG to Station ALOHA $\left(22^{\circ} 45^{\prime} \mathrm{N}, 158^{\circ} \mathrm{W}\right)$ in summer of 2015 , we collected samples within sunlit surface waters $(15 \mathrm{~m})$ every $4 \mathrm{~h}$ over the course of 8 days. We then conducted downstream lipidomic analyses to determine the concentrations of several major lipid classes (Fig. 1). A survey, conducted at a courser time resolution over 3 days in oligotrophic North Atlantic, suggested that small fluctuations in intact polar membrane lipids were possible in oligotrophic ocean environments ${ }^{18}$. The betaine lipid diacylglycerylcarboxy- $N$-hydroxymethyl-choline (DGCC) is an intact polar membrane lipid that is associated with eukaryotic phytoplankton in samples from the oligotrophic regions ${ }^{19}$. The time-of-day average (Fig. 1b) of DGCC was highest at dusk $(\sim 18: 00 \mathrm{~h}$ local time) and lowest at dawn $(\sim 06: 00 \mathrm{~h})$, but the $1.27 \pm 0.2$-fold difference was not significant $(t$-test, $t(\mathrm{df}=8)$ $=-1.947, p=0.0874)$. Furthermore, diel $(24-\mathrm{h})$ periodicity was not detected for concentrations of this lipid class $(p>0.05)$ using the RAIN (Rhythmicity Analysis Incorporating Nonparametric methods) algorithm ${ }^{20}$. DGCC is expected to scale nearly linearly with the number of cells ${ }^{21}$, and thus the relatively stable concentrations of DGCC at Station ALOHA indicates that the number of cells was also similarly invariant throughout the diel cycle of sunlight, i.e. production of cells was balanced by grazing and other mortality factors.

In contrast, TAG concentrations showed significant diel periodicity (RAIN algorithm, $p=0.004$ ) with dramatic oscillations over the diel cycle $(2.3 \pm 0.4$-fold mean $\pm \mathrm{SD}$; $t$-test, $\mathrm{t}(\mathrm{df}=8)=-6.200 ; p=0.0002)$. Highest abundances in TAGs occurred during the day near dusk, and lowest abundances at dawn. The significantly greater oscillations in TAG concentrations relative to DGCCs (and relative to particulate organic carbon (Supplementary Figure 1)) indicates that the TAG signal is not simply a reflection of cell size or abundance, but is instead consistent with the synthesis and accumulation of TAGs by cells during daylight hours and depletion at night. Eukaryotic phytoplankton have been proposed as the main source of TAGs in the ocean ${ }^{22}$ and the distribution of individual molecular species of TAGs is consistent with other reports from eukaryotic phytoplankton from the Pacific with a dominance of TAGs containing 48, 50, and 52 carbon atoms and one unsaturation ${ }^{22}$ (Supplementary Figure 2). To confirm that eukaryotic phytoplankton dominate the TAG signal in the NPSG, we screened several strains of Prochlorococcus and Pelagibacter, the numerically dominant genera of cyanobacteria and heterotrophic bacteria, respectively, in oligotrophic gyres ${ }^{23,24}$. These two groups account for more than half of the biomass in these regions ${ }^{25}$. No TAGs were detected in any of the strains, consistent with the lack of TAG biosynthesis genes in both groups. Other bacteria (e.g., from the genera Mycobacterium, Streptomyces, and Rhodococcus) known to produce $\mathrm{TAGs}^{25}$ are not abundant in surface waters of the NPSG ${ }^{26}$. By contrast, the eukaryotic phytoplankton in the oligotrophic gyres are dominated by haptophytes, dinoflagellates, diatoms, and prasinophytes 27,28 , and all of these groups have the ability to synthesize TAGs ${ }^{13}$. The relative magnitude of TAG increase we observed is in the range of what has been observed in culture experiments. In the haptophyte Isochrysis sp. grown under $\mathrm{N}$-limitation, neutral lipid concentrations, which were likely mostly TAGs, increased by $\sim 1.7$-fold between the beginning and end of the photoperiod ${ }^{29}$. Similarly, in the dinoflagellate coral symbiont Symbiodinium, the quantity of TAGs in lipid bodies increase by approximately twofold ${ }^{17}$. Thus, we concluded that the marked diel cycles in TAG concentrations we observed in the NPSG were most likely driven by eukaryotic phytoplankton. 

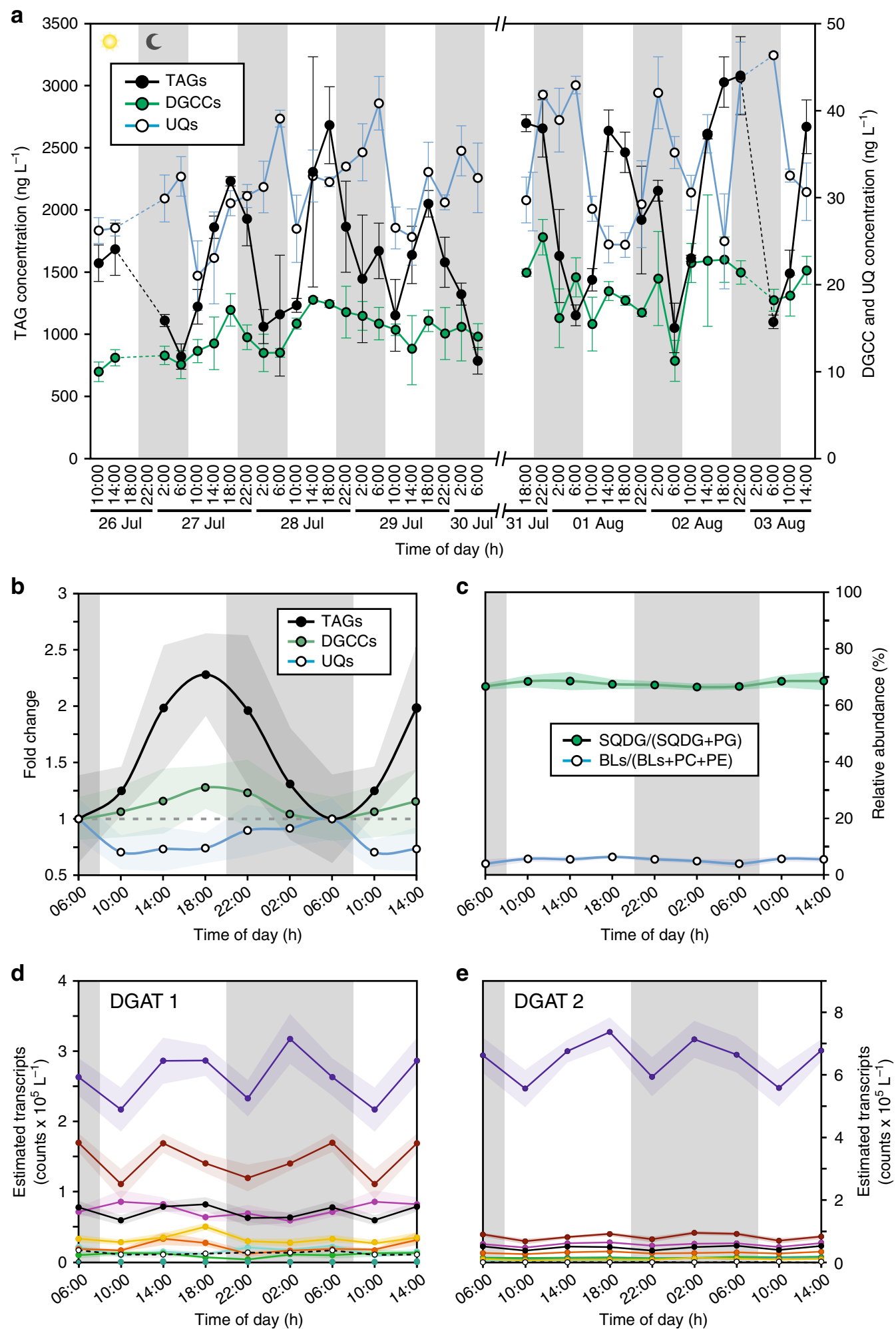

$$
\begin{aligned}
& \rightarrow \text { Dinophyceae } \rightarrow \text { Haptophyta } \rightarrow \text { Alveolata }^{*} \rightarrow \text { Opisthokonta } \rightarrow \text { Viridiplantae } \\
& \rightarrow \text { Cryptophyta } \rightarrow \text { Bacillariophyta } \rightarrow \text { - Heterokonta }
\end{aligned}
$$

Another class of lipids, the ubiquinones (UQs), also displayed a diel periodicity in concentration (RAIN algorithm, $p=0.002$ ), but the phase was opposite of TAGs with a pre-dawn maximum followed by a precipitous drop to an early-morning minimum $(0.3 \pm 0.1$-fold mean $\pm \mathrm{SD} ; t$-test, $t(\mathrm{df}=8)=3.616, p=0.0068)$ (Fig. 1a and b). UQs are essential components of the electron transport chain used for cellular respiration by most aerobic organisms $s^{30}$. In the NPSG, these lipids are likely derived primarily from eukaryotic microbes as cyanobacteria do not synthesize UQs - cyanobacteria use plastoquinones for photosynthetic electron transport and respiration ${ }^{31}$. Furthermore, we observed that the distribution of different UQ molecules in the 
Fig. 1 Diel oscillation of lipid biomarkers. a Concentrations of storage lipids (TAGs), a class of structural membrane lipids from eukaryotic phytoplankton (DGCCs) and lipid-soluble electron transporters (UQs) over 8 day/night cycles at $15 \mathrm{~m}$ water depth in July and August 2015 at Station ALOHA. Values represent the average of environmental triplicates and error bars the standard deviation. $\mathbf{b}$ Time-of-day average of fold change relative to 06:00 $\mathrm{h}$ (local time) of TAG, DGCC and UQ concentrations. c Ratios of intact non-phosphorus membrane lipids (SQDG and BLs) to membrane phospholipids (PG, PE, and PC). Lipid ratios are based on known lipid substitutions in eukaryotic phytoplankton and cyanobacteria under nutrient limitation ${ }^{42}$. $\mathbf{d}$ and $\mathbf{e}$ Time-of-day average of diacylglycerol acyltransferase 1 (DGAT1) and DGAT2 abundance, respectively, in major eukaryotic phytoplankton ( ${ }^{\star}$ not including Dinophyceae (dinoflagellates), ${ }^{\star \star}$ not including Bacillariophyta (diatoms)). Shaded areas around lines in $\mathbf{b}$ and $\mathbf{c}$ represent the standard deviation of averaged values for each time of day $(n \geq 6)$. Vertical gray shaded areas in each graph indicate night. TAG triacylglycerol; DGCC diacylglycerylcarboxy- $N$-hydroxymethylcholine, UQ ubiquinone; SQDG sulfoquinovosyl diacylglycerol; PG phosphatidylglycerol; BL betaine lipid (sum of DGCC, diacylglyceryl trimethylhomoserine (DGTS), and diacylglyceryl hydroxymethyl-trimethyl- $\beta$-alanine (DGTA)); PC phosphatidylcholine; PE phosphatidylethanolamine

NPSG differs from that of Pelagibacter (Supplementary Figure 3). Respiratory quinone concentrations in cultures and environmental samples have been suggested to reflect respiratory activity of their producers ${ }^{32-34}$ and the observed diel oscillations of UQs may indicate enhanced cellular respiration at night, which is consistent with the consumption of TAGs by phytoplankton. In addition, it has recently been proposed, based on genomic analysis, that increased ubiquinone levels might be required in Escherichia coli cells when grown on long-chain fatty acids ${ }^{35}$. Ubiquinones exhibit antioxidant properties and could mitigate elevated levels of reactive oxygen species generated by fatty acid degradation. That this strategy occurs in eukaryotic phytoplankton remains to be shown, but it could explain our observation of higher ubiquinone concentrations at night when TAGs are degraded.

Metatranscriptome analysis of the plankton community. Within the NPSG, the phytoplankton community is dominated by cyanobacterial and eukaryotic picophytoplankton cells less than $\sim 2-3 \mu \mathrm{m}$ and eukaryotic nanophytoplankton in the $2-20 \mu \mathrm{m}$ size range ${ }^{36}$. To identify which eukaryotic phytoplankton were the likely sources of TAGs in the NPSG, we analyzed metatranscriptome expression patterns of two genes that encode characterized diacylglycerol acyltransferase isoforms (DGAT1 and DGAT2), both of which commit the final step in TAG biosynthesis ${ }^{37}$. Thus, a transcriptional signal of these genes is expected for all cells producing TAGs regardless of how the observed diel cycle is regulated. In most eukaryotic algae, DGAT1 is encoded by a single-copy gene, whereas DGAT2 is often encoded by multiple gene copies ${ }^{38}$. DGAT1 is widely distributed across marine phytoplankton, with distinct clades of sequences that largely reflect taxonomic groupings. Notably, however, cultured isolates of the smallest and most abundant eukaryotic picophytoplankton, the prasinophytes Ostreococcus and Micromonas, lack DGAT1 and instead rely on multi-copy DGAT2 for TAG biosynthesis ${ }^{38}$. In the NPSG, haptophyte and dinoflagellate taxa dominate the DGAT1 transcriptome signal $\left(\sim 6.4 \times 10^{5}\right.$ counts $\left.\mathrm{L}^{-1}\right)$, together accounting for almost $70 \%$ of the detected DGAT1 transcripts (Table 1; Supplementary Figure 4), regardless of time of day. Despite the distinct diel pattern in TAG abundance, transcript abundance for the DGAT1 gene remained relatively constant throughout the four days of transcript sampling (Fig. 1d), suggesting that TAG abundance in the NPSG might be regulated through intracellular consumption instead of production. Dinoflagellates, which accounted for the majority of DGAT1 transcripts, are known for their lack of transcriptional regulation ${ }^{39}$ and an absence of transcriptional rhythmicity within the Haptophyta clade was also expected given that in culture, the haptophyte Chrysochromulina tobin, displays no diel oscillations in $D G A T 1^{40}$. We also examined transcript abundance associated with DGAT2 (Fig. 1e) to determine the role of prasinophytes in TAG biosynthesis. As with the DGAT1 gene, transcript

\section{Table 1 Relative abundance of DGAT1 transcripts in the} NPSG

Taxonomic group ${ }^{\mathbf{a}}$

Relative abundance $(\%)^{\mathrm{b}}$

Opisthokonts, glaucophytes, cryptophytes

Oikopleura dioica

Lepeophtheirus salmonis

6.4

Alveolates ${ }^{c}$

Strombidium inclinatum

Dinophyceae (dinoflagellates)

Ceratium fusus

Gymnodinium catenatum

Noctiluca scintillans

Scrippsiella trochoidea

Bolidomonas sp

Heterokonts $^{d}$

Viridiplantae (green algae)

Paraphysomonas imperforata

Haptophytes

Chrysochromulina polylepis

Chrysochromulina brevifilum

Bacillariophyta (diatoms)

1.1

2.3

4.4

3.5

42.5

8.8

9.6

2.4

1.4

4.4

12.1

1.4

1.0

25.3

1.3

11.8

3.2

aTaxonomic groups represent the best read placements in the maximum-likelihood phylogenetic tree constructed from publicly available marine genomes and transcriptomes (see

Supplementary Figure 4)

bRelative abundances for species within clades are given when recruited reads were $>1 \%$ of total reads

cNot including Dinophyceae

${ }^{\mathrm{d} N o t}$ including Bacillariophyta

abundance $\left(\sim 9.1 \times 10^{6}\right.$ counts $\left.\mathrm{L}^{-1}\right)$ for the DGAT2 gene across the different clades remained constant over the 4 days, although potential diel cycles for specific copies could be obscured by this analysis; culture studies with $C$. tobin indicate that only one of the nine DGAT2 gene copies displays a diel cycle in transcript abundance $^{40}$. The DGAT2 transcript signal was also dominated by dinoflagellate taxa with few (average $<1 \%$ ) transcripts detected for prasinophytes (Fig. 1e). A number of genes involved in the production of the precursors to TAGs, phosphatidic acids (e.g., acyltransferases) and in the consumption of TAGs (e.g., lipases) were also examined, and none showed regular diel variations, with the exception of a single, relatively scarce acyltransferase and a single acyl-CoA binding protein from haptophytes (Supplementary Table 1). Together the metatranscriptome data suggest that eukaryotic nanophytoplankton, particularly the haptophytes and dinoflagellates, dominate TAG production in the NPSG.

TAG production rates throughout the photic zone. In culture experiments, nutrient limitation can be the major stimulus for TAG accumulation in phytoplankton ${ }^{13}$ and the growth rate of eukaryotic phytoplankton in the NPSG has been shown to be limited by nutrients ${ }^{40,41}$. An archetypal microbial response to nutrient stress, particularly phosphorus stress, is the substitution of non-phosphorus membrane lipids for phospholipids; this 
response has been observed in nearly all of the major classes of plankton in the NPSG, including Prochlorococcus, Synechococcus, Pelagibacter, diatoms, heterokonts, and haptophytes ${ }^{21,42-44}$. Furthermore, we have previously shown that differences in the ratios of non-phosphorus lipids to phospholipids between the oligotrophic ocean ecosystems, including the NPSG, reflect differences in ambient phosphate concentrations and turnover rates ${ }^{42}$. These ratios of membrane lipids showed no significant diel variations over the investigated time interval (Fig. 1c), suggesting the absence of diel fluctuations in nutrient stress. In haptophytes, TAGs, along with diagnostic viral glycosphingolipids, have also been shown to accumulate in response to viral infection ${ }^{45}$, but these viral glycosphingolipids were not detected in any of the NPSG samples we analyzed. Thus, the diel TAG cycle in the NPSG was not likely a reflection of daily fluctuations in nutrient stress or viral mortality.

We posit instead that the magnitude of the diel signal in biosynthesis of TAGs is driven directly by sunlight availability. This is evidenced by depth profiles of net TAG concentrations collected at dusk and dawn in March 2016 (Fig. 2a), showing that the magnitude of diel periodicity observed in surface waters is dampened with depth and disappears below $100 \mathrm{~m}$, the depth where net photosynthetic carbon assimilation approaches zero (Fig. 2b). Accordingly, daily TAG production rates derived from these concentrations were highest in the surface waters $(0.42 \pm$ $0.15 \mathrm{mg}$ TAG $\mathrm{C} \mathrm{m}^{-3} \mathrm{~d}^{-1} ; n=6$ ) and decreased with water depth (Fig. 2b). The nitricline at Station ALOHA is typically at around $125 \mathrm{~m}^{46}$, and thus decreasing nutrient stress with depth cannot explain the observed decrease in TAG production rate. The invariant molecular distribution of TAGs across different water depths (Fig. 2c) further suggests a similar biological source of TAGs throughout the euphotic zone, which is consistent with the major nanophytoplankton groups, diatoms, haptophytes, and dinoflagellates, being found throughout these waters in similar abundances ${ }^{27}$. Accordingly, the molecular distribution of DGCCs was highly similar at different water depths and different times of the day (Supplementary Figures 2 and 5).

TAG contribution to primary production. To estimate the contribution of TAGs to daily biomass production, we calculated net TAG production rates based on the difference in TAG concentrations between dawn and dusk, and compared these rates with net primary production rates determined during the same time interval (Table 2). In cells of some eukaryotic phytoplankton in culture, including dinoflagellates and haptophytes, TAGs can account for up to $50 \%$ of the cellular dry weight ${ }^{13}$, illustrating the potential for a large investment of photosynthetic energy into TAG synthesis. The ${ }^{14} \mathrm{C}$-based primary productivity $\left({ }^{14} \mathrm{C}-\mathrm{PP}\right)$ of a

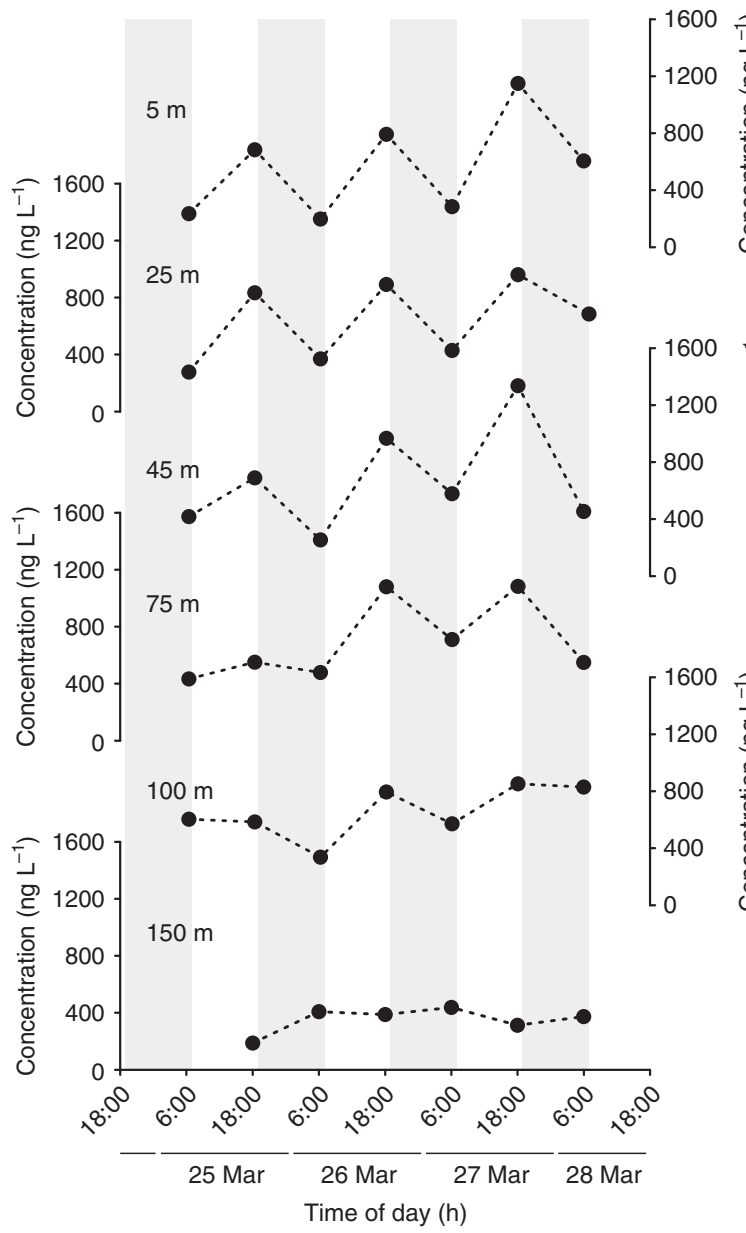

b

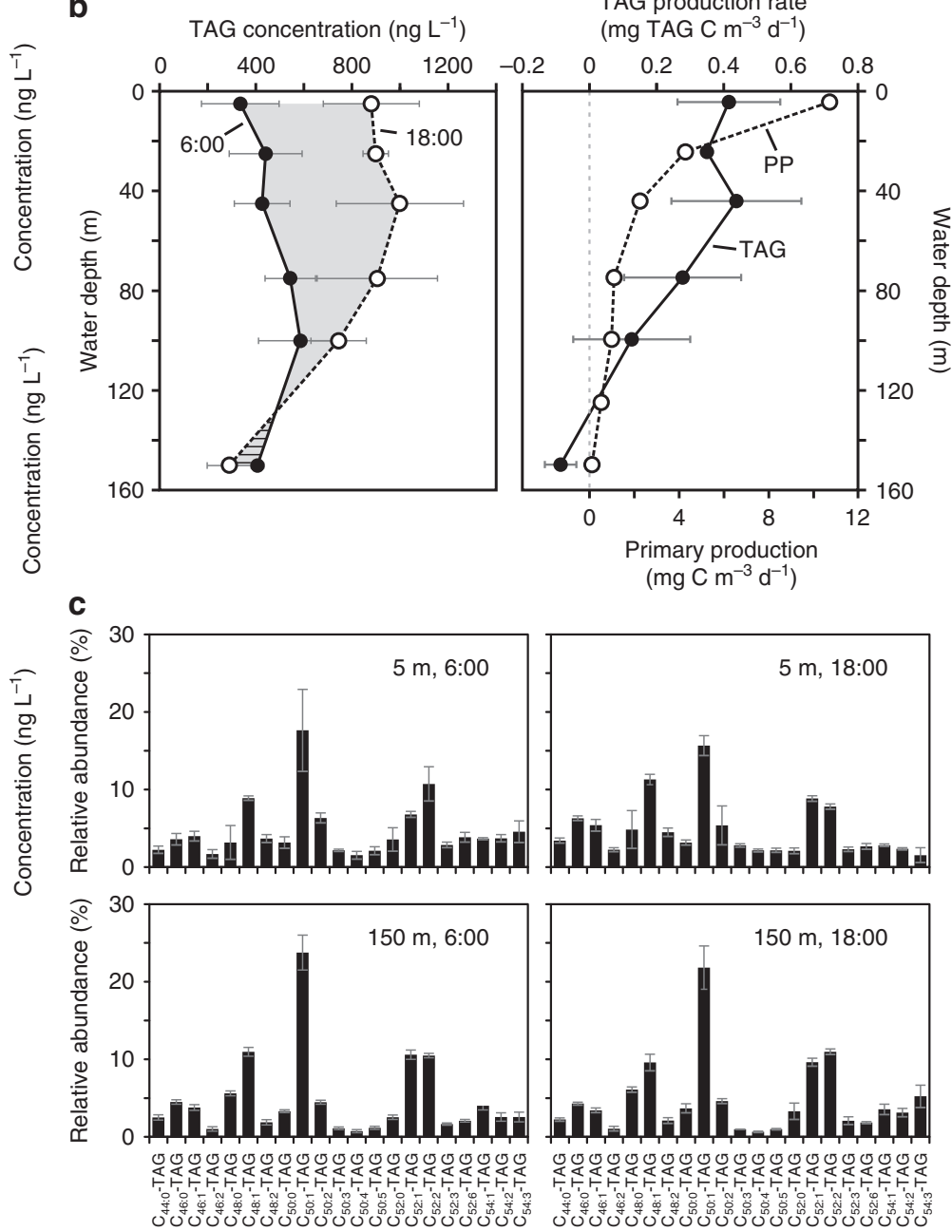

Fig. 2 Diel patterns in triacylglycerols at different water depths. a Concentrations of triacylglycerols (TAGs) for dusk ( 06:00 h, local time) and dawn ( 18:00 h) over 4 day/night cycles (gray areas in indicate night) in March 2016 at different water depths at Station ALOHA. b Time-of-day average of depth profiles of TAGs and derived production rates ( $\mathrm{mg} \mathrm{TAG} \mathrm{C} \mathrm{m}^{-3} \mathrm{~d}^{-1}$ ) as well as primary production (PP, mg C m $\mathrm{m}^{-3} \mathrm{~d}^{-1}$ ). c TAG composition averaged for time of day at 5 and $150 \mathrm{~m}$ water depth. Bars in $\mathbf{b}$ and $\mathbf{c}$ are the mean $\pm \mathrm{SD}(n \geq 3)$ 
Table 2 Triacylglycerol (TAG) production rates and their contribution to overall daytime net primary production (PP)

\begin{tabular}{|c|c|c|c|c|c|c|c|c|}
\hline \multirow[t]{3}{*}{ Summer 2015} & $7 / 27 / 2015$ & 15 & $17.6 \pm 0.4$ & $5.4 \pm 2$ & 7.6 & 1.08 & 6.1 & 20 \\
\hline & $7 / 29 / 2015$ & 15 & $16.9 \pm 1.5$ & $4.3 \pm 0.8$ & 6.2 & 0.69 & 4.1 & 16 \\
\hline & $8 / 1 / 2015$ & 15 & $14.7 \pm 1.2$ & $3.2 \pm 0.7$ & 6.1 & 1.13 & 7.7 & 36 \\
\hline \multirow[t]{2}{*}{ Spring 2016} & $3 / 25 / 2016$ & 25 & $5.8 \pm 0.5$ & n.d. & 1.8 & 0.42 & 7.3 & n.d. \\
\hline & $3 / 26 / 2016$ & 25 & $5.0 \pm 0.7$ & $2.7 \pm 1.0$ & 0.7 & 0.40 & 8.0 & 15 \\
\hline
\end{tabular}

the total NPSG community at $15 \mathrm{~m}$ in summer 2015 ranged between $14.7 \pm 2.4$ and $17.6 \pm 0.4 \mathrm{mg} \mathrm{C} \mathrm{m}^{-3} \mathrm{~d}^{-1}$, whereas primary production by nanophytoplankton $(2-20 \mu \mathrm{m}$ particle size fraction) was between $2.9 \pm 0.9$ and $5.4 \pm 2 \mathrm{mg} \mathrm{C} \mathrm{m}^{-3} \mathrm{~d}^{-1}$, which is as expected in the NPSG where Prochlorococcus is numerically dominant. The TAG production rate in surface waters $(15 \mathrm{~m})$ was $1.0 \pm 0.2 \mathrm{mg} \mathrm{C} \mathrm{m}^{-3} \mathrm{~d}^{-1}$ (mean $\left.\pm \mathrm{SD} ; n=4\right)$, which represented $6.4 \pm 1.7 \%$ of total daytime net primary production and $28 \pm 10 \%$ of primary production by nanophytoplankton. Similar results were found in the spring of 2016, when TAG synthesis averaged $0.41 \pm 0.01 \mathrm{mg} \mathrm{C} \mathrm{m}^{-3} \mathrm{~d}^{-1}(n=3)$ and was $7.5 \pm 0.5 \%$ of total daytime net primary production and $14 \pm 2 \%$ of nanoeukaryotic primary production. An estimate of the global significance of TAGs by assuming that the TAG production rates from the spring are representative of mean rates throughout the year at Station ALOHA, integrating them through the photic zone, and extending them to the total area of subtropical gyres (see Supplementary Methods for calculation), indicates that the daily accumulation and depletion of TAGs could drive a carbon flux

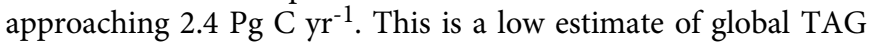
synthesis because this phenomenon likely also occurs outside the subtropical gyres in high latitude, equatorial, or coastal regions where primary production rates are higher and eukaryotic phytoplankton are more abundant. Thus, the total global percentage contribution of TAGs to primary production could be substantial, although light may limit carbon fixation in some of these other environments ${ }^{47,48}$.

\section{Discussion}

The diel cycle in TAG concentrations we observed in the NPSG is evidence that the biochemical composition and caloric content of eukaryotic phytoplankton changes profoundly throughout the course of a day. The availability of light energy cycles between extremes of darkness and abundance on a diel cycle, and phytoplankton buffer these variations by transforming this resource into stored chemical energy. Lipid molecules such as TAGs contain more chemical energy per unit mass than other classes of biochemicals, such as starch or carbohydrates ${ }^{11}$, and the caloric content of phytoplankton is strongly correlated to their lipid content ${ }^{49}$. TAG molecules contain only atoms of hydrogen, carbon, and oxygen, elements that are readily available from water and dissolved carbon dioxide; consequently, TAG synthesis by phytoplankton does not incur a stoichiometric requirement for other elements, such as nitrogen, that are scarce in their environments. Thus, TAGs are an efficient molecular vehicle for storing chemical energy that is suited to the environmental conditions experienced by phytoplankton in the NPSG.

Due to the significant daily changes in the TAG content of eukaryotic phytoplankton, the timing of their mortality could have a large impact on ocean carbon cycling in the NPSG. Using the same approach as Platt and Irwin ${ }^{49}$, we calculate that phytoplankton in the NPSG could contain as much as $40 \%$ more calories at dusk than at dawn (see Supplementary Methods for calculations). Thus, we hypothesize that eukaryotic phytoplankton would be more favorable prey for zooplankton at dusk than at dawn. Vertical mesozooplankton distributions in the NPSG and throughout the oceans show strong diel changes, with mesozooplankton ascending from the mesopelagic zone to the surface at dusk and descending at dawn. This daily migration, the largest coordinated movement of animals on the planet ${ }^{50}$, balances two competing objectives: rapid growth through efficient feeding in phytoplankton-rich surface waters, and minimization of risk of mortality from visual predators. Traditionally, the cue for diel vertical migration of mesozooplankton was thought to be a relative change in visible light ${ }^{51}$. However, diel vertical migration was also observed in deep sea plankton and it was hypothesized that a precise biochemical clock could maintain the solar diurnal rhythms in deep sea plankton motions ${ }^{52,53}$. We hypothesize, based on the evidence in this study, that the caloric enrichment of phytoplankton at night could provide a strong evolutionary reinforcement for diel vertical migration by mesozooplankton. Life cycles of some mesozooplankton species depend on the nutritional quality of phytoplankton, as defined by lipid content ${ }^{54}$.

In addition to potentially impacting the timing of zooplankton grazing, the diel fluctuations in TAG content of eukaryotic phytoplankton are likely tightly coupled to the fate of organic matter within the NPSG. If TAGs are respired entirely by phytoplankton, then their synthesis would not contribute to net primary production even though TAG production is a substantial fraction of gross production. Using a simple model, we estimate that around $70 \%$ of the TAGs produced during the day are consumed by eukaryotic phytoplankton themselves, whereas $30 \%$ is consumed by mortality processes (see Supplementary Methods, Supplementary Figure 11 and Supplementary Table 2 for model details). Grazing mortality diverts the energy in TAGs to higher trophic levels, whereas mortality via autolysis or viral lysis of TAG-rich eukaryotic phytoplankton results in the release of relatively hydrophobic, calorierich, and nutrient-poor molecules to the pool of dissolved organic matter that fuels heterotrophic bacteria. The consequences of these fluctuations for carbon export, dissolved organic matter composition, nutrient cycling, and ecosystem function are unconstrained. TAGs accumulate in distinct intracellular lipid droplets, the fate of which upon release from lytic cells is also unknown. Understanding the timing and the amount of organic matter transferred from TAG-producing phytoplankton to higher trophic levels and/or the dissolved organic carbon pool will elucidate the consequences of TAG cycling for the marine food web and carbon cycling. 


\section{Methods}

Sample collection and lipid analysis by HPLC-MS. Seawater samples were collected during $R / V$ Kilo Moana cruises KM1513 (July/August 2015) and KM1605 (March 2016) near Station ALOHA $\left(22^{\circ} 45^{\prime} \mathrm{N}, 158^{\circ} 00^{\prime} \mathrm{W}\right)$ in the oligotrophic North Pacific Subtropical Gyre using standard Niskin-type bottles. Triplicate samples were obtained from the bottles during the July cruise and single samples during the March cruise. Samples ( 2 L) were filtered using vacuum filtration (ca. $-200 \mathrm{~mm}$ $\mathrm{Hg}$ ) onto $47 \mathrm{~mm}$ diameter $0.2 \mu \mathrm{m}$ hydrophilic Durapore filters (Millipore) and immediately flash-frozen and stored at $-196^{\circ} \mathrm{C}$ until processing. Filters were extracted using a modified Bligh and Dyer extraction ${ }^{55}$ with DNP-PE- $\mathrm{C}_{16: 0} / \mathrm{C}_{16: 0^{-}}$ DAG (2,4-dinitrophenyl phosphatidylethanolamine diacylglycerol; Avanti Polar Lipids, Inc., Alabaster, AL) used as an internal standard. Lipids were analyzed by reverse phase high performance liquid chromatography (HPLC) mass spectrometry (MS) on an Agilent 1200 HPLC (Agilent, Santa Clara, CA, USA) coupled to a Thermo Q Exactive Orbitrap high resolution mass spectrometer (ThermoFisher Scientific, Waltham, MA, USA). HPLC and MS conditions are described in Collins et al. ${ }^{56}$ (modified after Hummel et al. ${ }^{57}$ ). In brief: $20 \mu \mathrm{L}$ injections of sample were made onto a C8 Xbridge HPLC column (particle size $5 \mu \mathrm{m}$, length $150 \mathrm{~mm}$, width $2.1 \mathrm{~mm}$; Waters Corp., Milford, MA, USA). Eluent A was water with $1 \% 1 \mathrm{M}$ ammonium acetate and $0.1 \%$ acetic acid. Eluent B was $70 \%$ acetonitrile, $30 \%$ isopropanol with $1 \% 1 \mathrm{M}$ ammonium acetate and $0.1 \%$ acetic acid. Gradient elution was performed at a constant flow rate of $0.4 \mathrm{~mL} \mathrm{~min}^{-1}$ using the following gradient: $45 \% \mathrm{~A}$ for $1 \mathrm{~min}$ to $35 \% \mathrm{~A}$ at $4 \mathrm{~min}$, then from $25 \% \mathrm{~A}$ to $11 \% \mathrm{~A}$ at $12 \mathrm{~min}$, then to $1 \% \mathrm{~A}$ at $15 \mathrm{~min}$ with an isocratic hold until $25 \mathrm{~min}$, and finally back to $45 \%$ A for 5 min column equilibration. ESI source settings were: Spray voltage, $4.5 \mathrm{kV}$ $(+), 3.0 \mathrm{kV}(-)$; capillary temperature, $150{ }^{\circ} \mathrm{C}$; sheath gas and auxiliary gas, both 21 (arbitrary units); heated ESI probe temperature, $350^{\circ} \mathrm{C}$. Mass data were collected in full scan while alternating between positive and negative ion modes. For each MS full scan, up to three $\mathrm{MS}^{2}$ experiments targeted the most abundant ions with $\mathrm{N}_{2}$ as collision gas. A scan range of $100-1500 \mathrm{~m} / \mathrm{z}$ was used for all modes in sequence. The mass spectrometer was set to a resolving power of 140,000 (FWHM at $\mathrm{m} / \mathrm{z}$ 200) leading to an observed resolution of 75,100 at $\mathrm{m} / \mathrm{z} 875.5505$ of our internal standard, DNP-PE. Exact mass calibration was performed by weekly infusing a tune mixture. Additionally, every spectrum was corrected using a lock mass, providing real-time calibrations.

For the discovery, annotation, and putative identification of lipids in NPSG plankton, we used LOBSTAHS, an open-source lipidomics software pipeline that enables rapid identification of more than 20,000 individual lipids from an onboard library ${ }^{56}$ based on adduct ion abundances and several other orthogonal criteria (see Supplementary Data 1 for identified and annotated mass features by LOBSTAHS). Lipids identified using the LOBSTAHS software were quantified from MS data after pre-processing with $\mathrm{xcms}^{58}$ and CAMERA ${ }^{59}$. XCMS peak detection was validated by manual identification using retention time as well as accurate molecular mass and isotope pattern matching of proposed sum formulas in full-scan mode and tandem MS $\left(\mathrm{MS}^{2}\right)$ fragment spectra of representative compounds (Supplementary Figure 6).

Lipid quantification. Absolute lipid concentrations were calculated from peak areas of molecular ions in mass chromatograms using response factors from external standard curves. Individual response factors were determined by triplicate injection of a series of standard solutions in amounts ranging from $0.15 \mathrm{pmol}$ to 40 pmol on column. Standards for phosphatidylglycerol (PG), phosphatidylethanolamine (PE) and phosphocholine (PC), diacylglyceryl trimethylhomoserine (DGTS), and DNP-PE all contained two palmitic acid acyl groups and were purchased from Avanti Polar Lipids, Inc., (Alabaster, AL; USA). The abundances of diacylglyceryl hydroxymethyl-trimethyl- $\beta$-alanine (DGTA) and diacylglycerylcarboxy- $N$-hydroxymethyl-choline (DGCC) were corrected using the DGTS standard. Purified sulfoquinovosyl diacylglycerol (SQDG) from spinach was purchased from Lipid Products (South Nutfield, UK). Response factors for quinones were determined using a ubiquinone $\left(\mathrm{UQ}_{10: 10}\right)$ standard from Sigma-Aldrich (St. Louis, MO, USA). Triacylglycerols (TAGs) were quantified using a series of individual standards from Nu-Check-Prep, Inc. (Elysian, MN, USA). TAG response factors were based on the equivalent carbon number of each TAG after Holčapek et al. ${ }^{60}$ (Supplementary Figure 7). To validate peak integration by the XCMS software, we compared concentrations of selected lipids obtained from auto integration with manual integration (Supplementary Figure 8). Statistical significance of diel (24-h) rhythmicity of lipid data was determined using the RAIN nonparametric test in $\mathrm{R}^{20}$ with the default settings (independent).

Ion suppression was tested by further HPLC analysis of 30 selected samples spiked with the following deuterated standards: 1-pentadecanoyl-2-oleoyl(d7)-snglycero-3-phosphoethanolamine, 1,2-dipalmitoyl-sn-glycero-3-O-4'-[N,N,Ntrimethyl(d9)]-homoserine, D-glucosyl- $\beta-1,1^{\prime}$-N-stearoyl-D-erythro-sphingosined5, 1-hexadecanoyl-2-(9Z-octadecenoyl)-sn-glycero-3-phospho-(1'-rac-glycerol$\left.1^{\prime}, 1^{\prime}, 2^{\prime}, 3^{\prime}, 3^{\prime}-\mathrm{d} 5\right)$, and 1,3(d5)-dihexadecanoyl-2-octadecanoyl-glycerol, purchased from Avanti Polar Lipids, Inc., (Alabaster, AL; USA). No systematic matrix ion suppression was observed in these selected samples (Supplementary Figures 9 and 10 ) and thus the remaining $\approx 120$ samples were not similarly analyzed.

Transcriptomic analysis. Samples for transcriptomic analysis were collected during R/V Kilo Moana cruise KM1513 in tandem with the lipid samples.
Duplicate samples were sequentially filtered through a $100 \mu \mathrm{m}$ nylon mesh prefilter followed by a $142 \mathrm{~mm} 0.2 \mu \mathrm{m}$ polycarbonate filter using a peristaltic pump. Filters were flash frozen in liquid nitrogen and subsequently stored at $-80^{\circ} \mathrm{C}$ until further processing. Filters were extracted using the ToTALLY RNA Kit (Invitrogen) with some modifications. Briefly, frozen filters were added to $50 \mathrm{~mL}$ falcon tubes containing $5 \mathrm{~mL}$ of denaturation solution and extraction beads $(125 \mu \mathrm{L} 100$ $\mu \mathrm{m}$ zirconia beads, $125 \mu \mathrm{L} 500 \mu \mathrm{m}$ zirconia beads, and $250 \mu \mathrm{L} 425-600 \mu \mathrm{m}$ silica glass beads). In addition, to generate quantitative transcript inventories, a set of 14 internal mRNA standards were also added to the extraction buffer; these standards were synthesized according to Satinski et al. ${ }^{61}$ with the exception that eight standards were synthesized with poly(A) tails to mimic eukaryotic mRNAs. Total extracted RNA was treated with DNase I (Ambion, New York, USA) and purified with DNase inactivation reagent (Ambion). Purified total RNA was used to construct TruSeq cDNA libraries according to the Illumina TruSeq ${ }^{\circledast}$ RNA Sample Preparation v2 Guide. Briefly, RNAs were poly(A)-selected, converted to cDNAs, and sheared to $\sim 225 \mathrm{bp}$ fragments which were used to prepare cDNA libraries for paired-end $(2 \times 150)$ sequencing using the Illumina NextSeq 500 sequencing platform (Illumina Inc., San Diego, CA). Raw Illumina data were quality controlled with trimmomatic v0.36 ${ }^{62}$ using the parameters MAXINFO:135:0.5, LEADING:3, TRAILING:3, MINLEN:60, and AVGQUAL:20, and matching read pairs were merged using flash v1.2.11 ${ }^{63}$ with parameters -r 150 -f 250 -s 25. FASTQ files were converted to FASTA and translated with seqret and transeq vEMBOSS:6.6.0.0 $0^{64}$ using Standard Genetic Code, keeping only potential ORFs with length $>40$ amino acids.

Full-length DGAT1 and DGAT2 protein sequences from Arabidopsis thaliana (NP 179535; DGAT1) (NP 566952; DGAT2), Saccharomyces cerevisiae (NP_014888; DGAT2), Phaeodactylum tricornutum (43469; DGAT2), and Ostreococcus tauri (34368; DGAT2) were used as queries to identify orthologs in marine eukaryotic genomes and transcriptome assemblies available through Joint Genome Institute (JGI) and the Marine Microbial Eukaryote Transcriptome Sequence Project (MMETSP) ${ }^{65}$, respectively, using BLASTp. These included both experimentally verified and putative DGAT1/2 orthologs previously described ${ }^{38}$. Sequences were clustered using usearch at an identity of $80 \%$ (DGAT1) or $50 \%$ (DGAT2) and aligned using MAFFT (Version 7) with the E-ISN-I algorithm ${ }^{66}$ The alignment was trimmed using trimAl v1.267 using the -gt .05 -resoverlap 0.5 -seqoverlap 50 options, and any sequences that did not contain the highly conserved DGAT1 histidine residue were removed ${ }^{68}$. The trimmed alignment file was converted to Phylip format, and the best-fit amino acid substitution matrix (LG), among-site rate heterogeneity model (gamma distribution with invariable sites), and observed amino acid frequency were determined using ProtTest 3 software ${ }^{69,70}$. Outgroups included several related sterol O-acyltransferase protein sequences. A bootstrapped $(n=100)$, best-scoring $(n=10)$ maximum-likelihood tree was built using RAxML Version $8^{71,72 \text {, }}$

To recruit environmental metatranscriptomic reads to the DGAT1/2 reference alignments, an $\mathrm{hmm}$ profile was constructed from each reference alignment using hmmbuild followed by transcript identification and alignment using hmmsearch (parameters: - T 35 -incT 35) and hmmalign, respectively, using the HMMER package v3.1b2 $2^{73}$. Taxonomy was assigned to each environmental sequence using pplacer v1.1.alpha19-0-g807f6f ${ }^{74}$ based on the read placement with the best maximum likelihood score to the reference tree (parameters: --keep-at-most 1) Read counts for each edge were normalized by recovery of internal mRNA standards to estimate natural transcript abundance per liter of seawater ${ }^{61}$. To assess transcription of additional protein families, we obtained hmm profiles from Pfam $31.0^{75}$ for the protein families Acyltransferase (PF01553), Triglyceride lipase (PF01764), Lipase maturation factor (PF06762), and Acyl-CoA-binding protein (PF00887). These hmm profiles were then used with hmmsearch using the same parameters listed above. Statistical significance for diel periodicity of transcripts was tested using the RAIN package in $\mathrm{R}^{20}$

Primary production (PP) measurements. Standard Hawaii Ocean Time-series protocols were used to measure in vitro dawn to dusk PP via the ${ }^{14} \mathrm{C}$ radiotracer method $^{46}$. Water samples were collected from $25 \mathrm{~m}$ before dawn and incubated on deck from local dawn to dusk in incubators screened to $55 \%$ of surface irradiance and plumbed with flow through surface seawater for temperature regulation. Rate measurements in light bottles were corrected to remove the rate measured in parallel dark bottles, which were on average $4 \pm 1 \%(n=14)$ of light values. To determine night-time respiration, samples were incubated for $12 \mathrm{~h}$ in the light with ${ }^{14} \mathrm{C}$-labeled inorganic carbon and then incubated for a further $12 \mathrm{~h}$ in the dark as described in Marra and Barber ${ }^{76}$

The potential contributions of the $2-20 \mu \mathrm{m}$ size class to overall PP was calculated using an optical approach to measure particle size distributions (PSD) as described in White et al. ${ }^{77}$. Briefly, an in situ laser diffractometer (LISST- $\times 100$, Laser In Situ Scatterometer/Transmissometer, Sequoia Scientific Inc., hereafter simply LISST), was used to make continuous underway measurements of the PSD. Water was supplied from the ship's uncontaminated seawater, which drew seawater from $\sim 7 \mathrm{~m}$ below the waterline of the forward hull. For the first $10 \mathrm{~min}$ of every hour incoming seawater was passed through a $0.2 \mu \mathrm{m}$ filter. This dissolved blank was used to correct whole water signals collected in the succeeding 50 min of each hour. Particle volume estimated via a spherical inversion of forward scatter in 32 log-spaced bins ${ }^{77,78}$ was converted to carbon using the allometric scaling of 
Menden-Deuer and Lessard ${ }^{79}$ for non-diatom protistan plankton. Finally, the production of the 2-20 $\mu \mathrm{m}$ size class, which showed distinct diel cycles with a minimum at dawn and a maximum at dusk (Supplementary Figure 1), was calculated as the amplitude of this daily increase divided by the length of the photoperiod just as was done for TAG production.

The PP vertical profile for March 2016 was calculated by discretization of the below equation at the standard sampling depths of Station ALOHA: $\mathrm{P}\left(\mathrm{mg} \mathrm{C} \mathrm{m}^{-3}\right)$ $=12,000 \times \mathrm{PAR} \times[\mathrm{chl} a] \times a^{*} \times \Phi \mathrm{c}$, where 12,000 converts mol of carbon (C) to mg C, mean chlorophyll-specific absorption coefficient based on HPLC $\left(a^{*}, 0.026\right)$ is as reported by Letelier et al. ${ }^{80}$ for summer months and $\Phi c$, the quantum yield of photosynthesis [mol C mol quanta ${ }^{-1}$ ] was parameterized from ${ }^{14} \mathrm{C}$-based photosynthesis-irradiance curves as per Li et al. ${ }^{81}$.

\section{Data availability}

Sequence data are available in the NCBI BioProject database (accession number PRJNA396249). All other relevant data are available from the corresponding author upon request.

Received: 23 April 2018 Accepted: 25 October 2018

Published online: 05 December 2018

\section{References}

1. Poretsky, R. S. et al. Comparative day/night metatranscriptomic analysis of microbial communities in the North Pacific subtropical gyre. Environ. Microbiol. 11, 1358-1375 (2009).

2. Ottesen, E. A. et al. Multispecies diel transcriptional oscillations in open ocean heterotrophic bacterial assemblages. Science 345, 207-212 (2014).

3. Smith, S. R. et al. Transcriptional orchestration of the global cellular response of a model pennate diatom to diel light cycling under iron limitation. PLoS Genet. 12, e1006490 (2016).

4. Emerson, S. et al. Experimental determination of the organic carbon flux from open-ocean surface waters. Nature 389, 951-954 (1997).

5. Sarmiento, J. L. et al. Response of ocean ecosystems to climate warming. Glob. Biogeochem. Cycles 18, GB3003 (2004).

6. Wyman, M. \& Thom, C. Temporal orchestration of glycogen synthase (GlgA) gene expression and glycogen accumulation in the oceanic Picoplanktonic Cyanobacterium Synechococcus sp. Strain WH8103. Appl. Environ. Microbiol. 78, 4744-4747 (2012).

7. Halsey, K. H. \& Jones, B. M. Phytoplankton strategies for photosynthetic energy allocation. Ann. Rev. Mar. Sci. 7, 265-297 (2015).

8. Radakovits, R., Jinkerson, R. E., Darzins, A. \& Posewitz, M. C. Genetic engineering of algae for enhanced biofuel production. Eukaryot. Cell 9 , 486-501 (2010).

9. Diamond, S., Jun, D., Rubin, B. E. \& Golden, S. S. The circadian oscillator in Synechococcus elongatus controls metabolite partitioning during diurnal growth. Proc. Natl Acad. Sci. USA 112, E1916-E1925 (2015).

10. Lopez, J. S., Garcia, N. S., Talmy, D. \& Martiny, A. C. Diel variability in the elemental composition of the marine cyanobacterium Synechococcus. J. Plankton Res. 38, 1052-1061 (2016).

11. Berg, J. M., Tymoczko, J. L. \& Stryer, L. Biochemistry. Ch. 22 (W.H. Freeman, New York, 2002).

12. Talmy, D. et al. Flexible C: $\mathrm{N}$ ratio enhances metabolism of large phytoplankton when resource supply is intermittent. Biogeosciences 11, 4881-4895 (2014).

13. $\mathrm{Hu}$, Q. et al. Microalgal triacylglycerols as feedstocks for biofuel production: perspectives and advances. Plant J. 54, 621-639 (2008).

14. Müller-Navarra, D. C., Brett, M. T., Liston, A. M. \& Goldman, C. R. A highly unsaturated fatty acid predicts carbon transfer between primary producers and consumers. Nature 403, 74 (2000).

15. Müller-Navarra, D. C. et al. Unsaturated fatty acid content in seston and tropho-dynamic coupling in lakes. Nature 427, 69 (2004).

16. Boëchat, I. G. \& Giani, A. Seasonality affects diel cycles of seston biochemical composition in a tropical reservoir. J. Plankton Res. 30, 1417-1430 (2008).

17. Chen, H.-K. et al. Coral lipid bodies as the relay center interconnecting dieldependent lipidomic changes in different cellular compartments. Sci. Rep. 7, 3244 (2017).

18. Fulton, J. M., Fredricks, H. F. \& Van Mooy, B. A. S. Intact polar lipid export in the temperate western North Atlantic and Sargasso Sea. Org. Geochem. 114, 45-56 (2017).

19. Van Mooy, B. A. S. \& Fredricks, H. F. Bacterial and eukaryotic intact polar lipids in the eastern subtropical South Pacific: Water-column distribution, planktonic sources, and fatty acid composition. Geochim. Cosmochim. Acta 74, 6499-6516 (2010).
20. Thaben, P. F. \& Westermark, P. O. Detecting rhythms in time series with RAIN. J. Biol. Rhythms 29, 391-400 (2014).

21. Martin, P., Van Mooy, B. A. S., Heithoff, A. \& Dyhrman, S. T. Phosphorus supply drives rapid turnover of membrane phospholipids in the diatom Thalassiosira pseudonana. ISME J. 5, 1057 (2010).

22. Wakeham, S. G. \& Canuel, E. A. Organic geochemistry of particulate matter in the eastern tropical North Pacific Ocean: implications for particle dynamics. J. Mar. Res. 46, 183-213 (1988).

23. Campbell, L., Nolla, H. A. \& Vaulot, D. The importance of Prochlorococcus to community structure in the central North Pacific Ocean. Limnol. Oceanogr. 39, 954-961 (1994).

24. Eiler, A., Hayakawa, D. H., Church, M. J., Karl, D. M. \& Rappé, M. S. Dynamics of the SAR11 bacterioplankton lineage in relation to environmental conditions in the oligotrophic North Pacific subtropical gyre. Environ. Microbiol. 11, 2291-2300 (2009).

25. Alvarez, H. \& Steinbüchel, A. Triacylglycerols in prokaryotic microorganisms. Appl. Microbiol. Biotechnol. 60, 367-376 (2002).

26. DeLong, E. F. et al. Community genomics among stratified microbial assemblages in the ocean's interior. Science 311, 496-503 (2006).

27. Venrick, E. L. Phytoplankton species structure in the central North Pacific 1973-1996: variability and persistence. J. Plankton Res. 21, 1029-1042 (1999).

28. Simmons, M. P. et al. Abundance and biogeography of picoprasinophyte ecotypes and other phytoplankton in the Eastern North Pacific Ocean. Appl. Environ. Microbiol. 82, 1693-1705 (2016).

29. Lacour, T., Sciandra, A., Talec, A., Mayzaud, P. \& Bernard, O. Diel variations of carbohydrates and neutral lipids in nitrogen-sufficient and nitrogen-starved cyclostat cultures of isochrysis sp. 1. J. Phycol. 48, 966-975 (2012).

30. Collins, M. D. \& Jones, D. Distribution of isoprenoid quinone structural types in bacteria and their taxonomic implications. Microbiol. Rev. 45, 316-354 (1981).

31. Nowicka, B. \& Kruk, J. Occurrence, biosynthesis and function of isoprenoid quinones. Biochim. Biophys. Acta 1797, 1587-1605 (2010).

32. Elling, F. J. et al. Respiratory quinones in Archaea: phylogenetic distribution and application as biomarkers in the marine environment. Environ. Microbiol. 18, 692-707 (2016).

33. Hurley, S. J. et al. Influence of ammonia oxidation rate on thaumarchaeal lipid composition and the TEX ${ }_{86}$ temperature proxy. Proc. Natl Acad. Sci. USA 113, 7762-7767 (2016).

34. Becker, K. W. et al. Isoprenoid quinones resolve the stratification of microbial redox processes in a biogeochemical continuum from the photic zone to deep anoxic sediments of the Black Sea. Appl. Environ. Microbiol. 84, e2736-17 (2018).

35. Agrawal, S. et al. A genome-wide screen in Escherichia coli reveals that ubiquinone is a key antioxidant for metabolism of long chain fatty acids. J. Biol. Chem. 292, 20086-20099 (2017).

36. Takahashi, M. \& Bienfang, P. K. Size structure of phytoplankton biomass and photosynthesis in subtropical Hawaiian waters. Mar. Biol. 76, 203-211 (1983).

37. Yen, C.-L. E., Stone, S. J., Koliwad, S., Harris, C. \& Farese, R. V. DGAT enzymes and triacylglycerol biosynthesis. J. Lipid Res. 49, 2283-2301 (2008).

38. Chen, J. E. \& Smith, A. G. A look at diacylglycerol acyltransferases (DGATs) in algae. J. Biotechnol. 162, 28-39 (2012).

39. Alexander, H. et al. Functional group-specific traits drive phytoplankton dynamics in the oligotrophic ocean. Proc. Natl Acad. Sci. USA 112, E5972-E5979 (2015).

40. Hovde, B. T. et al. Genome sequence and transcriptome analyses of Chrysochromulina tobin: Metabolic tools for enhanced algal fitness in the prominent order Prymnesiales (Haptophyceae). PLoS Genet. 11, e1005469 (2015).

41. Moore, C. M. et al. Processes and patterns of oceanic nutrient limitation. Nat. Geosci. 6, 701-710 (2013).

42. Van Mooy, B. A. S. et al. Phytoplankton in the ocean use non-phosphorus lipids in response to phosphorus scarcity. Nature 458, 69-72 (2009).

43. Carini, P. et al. SAR11 lipid renovation in response to phosphate starvation. Proc. Natl Acad. Sci. USA 112, 7767-7772 (2015).

44. Cañavate, J. P., Armada, I. \& Hachero-Cruzado, I. Interspecific variability in phosphorus-induced lipid remodelling among marine eukaryotic phytoplankton. New Phytol. 213, 700-713 (2017).

45. Hunter, J. E., Frada, M. J., Fredricks, H. F., Vardi, A. \& Van Mooy, B. A. S. Targeted and untargeted lipidomics of Emiliania huxleyi viral infection and life cycle phases highlights molecular biomarkers of infection, susceptibility, and ploidy. Front. Mar. Sci. 2, 81 (2015).

46. Karl, D. M. \& Lukas, R. The Hawaii Ocean Time-series (HOT) program: background, rationale and field implementation. Deep Sea Res. Part II Top. Stud. Oceanogr. 43, 129-156 (1996).

47. Krishnamurthy, A., Moore, J. K., Zender, C. S. \& Luo, C. Effects of atmospheric inorganic nitrogen deposition on ocean biogeochemistry. J. Geophys. Res. 112, https://doi.org/10.1029/2006JG000334 (2007). 
48. Cloern, J. E. The relative importance of light and nutrient limitation of phytoplankton growth: a simple index of coastal ecosystem sensitivity to nutrient enrichment. Aquat. Ecol. 33, 3-15 (1999).

49. Platt, T. \& Irwin, B. Caloric content of phytoplankton. Limnol. Oceanogr. 18, 306-310 (1973).

50. Cushing, D. H. The vertical migration of planktonic Crustacea. Biol. Rev. 26, 158-192 (1951).

51. Gliwicz, M. Z. Predation and the evolution of vertical migration in zooplankton. Nature 320, 746 (1986)

52. van Haren, H. \& Compton, T. J. Diel vertical migration in deep sea plankton is finely tuned to latitudinal and seasonal day length. PLOS ONE 8, e64435 (2013).

53. Häfker, N. S. et al. Circadian clock involvement in Zooplankton diel vertical migration. Curr. Biol. 27, 2194-2201.e3 (2017).

54. Søreide, J. E., Leu, E., Berge, J., Graeve, M. \& Falk-Petersen, S. Timing of blooms, algal food quality and Calanus glacialis reproduction and growth in a changing Arctic. Glob. Chang. Biol. 16, 3154-3163 (2010).

55. Popendorf, K. J., Fredricks, H. F. \& Van Mooy, B. A. S. Molecular ionindependent quantification of polar glycerolipid classes in marine plankton using triple quadrupole MS. Lipids 48, 185-195 (2013).

56. Collins, J. R., Edwards, B. R., Fredricks, H. F. \& Van Mooy, B. A. S. LOBSTAHS: an adduct-based lipidomics strategy for discovery and identification of oxidative stress biomarkers. Anal. Chem. 88, 7154-7162 (2016).

57. Hummel, J. et al. Ultra performance liquid chromatography and high resolution mass spectrometry for the analysis of plant lipids. Front. Plant Sci. 2, 54 (2011).

58. Smith, C. A., Want, E. J., O’Maille, G., Abagyan, R. \& Siuzdak, G. XCMS: processing mass spectrometry data for metabolite profiling using nonlinear peak alignment, matching, and identification. Anal. Chem. 78, 779-787 (2006).

59. Kuhl, C., Tautenhahn, R., Böttcher, C., Larson, T. R. \& Neumann, S. CAMERA: an integrated strategy for compound spectra extraction and annotation of LC/MS data sets. Anal. Chem. 84, 283-289 (2012).

60. Holčapek, M., Lísa, M., Jandera, P. \& Kabátová, N. Quantitation of triacylglycerols in plant oils using HPLC with APCI-MS, evaporative lightscattering, and UV detection. J. Sep. Sci. 28, 1315-1333 (2005).

61. Satinsky, B. M., Gifford, S. M., Crump, B. C. \& Moran, M. A. Use of internal standards for quantitative metatranscriptome and metagenome analysis. Methods Enzymol. 531, 237-250 (2013).

62. Bolger, A. M., Lohse, M. \& Usadel, B. Trimmomatic: a flexible trimmer for Illumina sequence data. Bioinformatics 30, 2114-2120 (2014).

63. Magoč, T. \& Salzberg, S. L. FLASH: fast length adjustment of short reads to improve genome assemblies. Bioinformatics 27, 2957-2963 (2011).

64. Rice, P., Longden, I. \& Bleasby, A. EMBOSS: the European Molecular Biology Open Software Suite. Trends Genet. 16, 276-277 (2000).

65. Keeling, P. J. et al. The Marine Microbial Eukaryote Transcriptome Sequencing Project (MMETSP): illuminating the functional diversity of eukaryotic life in the oceans through transcriptome sequencing. PLoS Biol. 12, e1001889 (2014)

66. Katoh, K. \& Standley, D. M. MAFFT multiple sequence alignment software version 7: improvements in performance and usability. Mol. Biol. Evol. 30, 772-780 (2013).

67. Capella-Gutiérrez, S., Silla-Martínez, J. M. \& Gabaldón, T. trimAl: a tool for automated alignment trimming in large-scale phylogenetic analyses. Bioinformatics 25, 1972-1973 (2009).

68. McFie, P. J., Stone, S. L., Banman, S. L. \& Stone, S. J. Topological orientation of acyl-CoA:Diacylglycerol acyltransferase-1 (DGAT1) and identification of a putative active site histidine and the role of the $\mathrm{N}$ terminus in dimer/tetramer formation. J. Biol. Chem. 285, 37377-37387 (2010).

69. Darriba, D., Taboada, G. L., Doallo, R. \& Posada, D. ProtTest 3: fast selection of best-fit models of protein evolution. Bioinformatics 27, 1164-1165 (2011).

70. Guindon, S. \& Gascuel, O. A simple, fast, and accurate algorithm to estimate large phylogenies by maximum likelihood. Syst. Biol. 52, 696-704 (2003)

71. Stamatakis, A., Hoover, P. \& Rougemont, J. A rapid bootstrap algorithm for the RAxML Web servers. Syst. Biol. 57, 758-771 (2008).

72. Stamatakis, A. RAxML version 8: A tool for phylogenetic analysis and postanalysis of large phylogenies. Bioinformatics 30, 1312-1313 (2014).

73. Eddy, S. R. Profile hidden Markov models. Bioinformatics 14, 755-763 (1998).
74. Matsen, F. A., Kodner, R. B. \& Armbrust, E. V. pplacer: linear time maximum likelihood and Bayesian phylogenetic placement of sequences onto a fixed reference tree. BMC Bioinform. 11, 538 (2010).

75. Finn, R. D. et al. The Pfam protein families database: towards a more sustainable future. Nucleic Acids Res. 44, D279-D285 (2016).

76. Marra, J. \& Barber, R. T. Phytoplankton and heterotrophic respiration in the surface layer of the ocean. Geophys. Res. Lett. 31, L09314 (2004)

77. White, A. E. et al. Phenology of particle size distributions and primary productivity in the North Pacific subtropical gyre (Station ALOHA). J. Geophys. Res. Ocean 120, 7381-7399 (2015).

78. Barone, B. et al. Particle distributions and dynamics in the euphotic zone of the North Pacific Subtropical Gyre. J. Geophys. Res. Ocean 120, 3229-3247 (2015).

79. Menden-Deuer, S. \& Lessard, E. J. Carbon to volume relationships for dinoflagellates, diatoms, and other protist plankton. Limnol. Oceanogr. 45, 569-579 (2000).

80. Letelier, R. M. et al. Light absorption by phytoplankton in the North Pacific Subtropical Gyre. Limnol. Oceanogr. 62, 1526-1540 (2017).

81. Li, B., Karl, D. \& Letelier, R. M. Size-dependent photosynthetic variability in the North Pacific Subtropical Gyre. Mar. Ecol. Prog. Ser. 440, 27-40 (2011).

\section{Acknowledgements}

We are grateful to the officers and crew of the $R / V$ Kilo Moana cruises KM1513 and KM1605. We further thank Allison Coe in the Chisholm Lab at MIT for providing Prochlorococcus biomass. Thanks to four anonymous reviewers whose comments greatly improved this manuscript. This work was supported by a grant from the Simons Foundation, and is a contribution of the Simons Collaboration on Ocean Processes and Ecology (SCOPE award \# 329108, B.A.S.V.M.). K.W.B. was further supported by the Postdoctoral Scholarship Program at Woods Hole Oceanographic Institution \& U.S Geological Survey.

\section{Author contributions}

K.W.B., lipidomics, data analysis, and paper writing; B.A.S.V.M, study design, paper writing; J.R.C., lipidomics and data analysis; B.P.D., R.D.G. and E.V.A., transcriptomics and data analysis. A.E.W., primary productivity measurements. H.F.F., lipidomics and data analysis; D.J.R. and J.E.O., onboard sampling; P.C., Pelagibacter biomass provision; All authors provided editorial comments on the manuscript.

\section{Additional information}

Supplementary Information accompanies this paper at https://doi.org/10.1038/s41467018-07346-z.

Competing interests: The authors declare no competing interests.

Reprints and permission information is available online at http://npg.nature.com/ reprintsandpermissions/

Publisher's note: Springer Nature remains neutral with regard to jurisdictional claims in published maps and institutional affiliations.

Open Access This article is licensed under a Creative Commons Attribution 4.0 International License, which permits use, sharing, adaptation, distribution and reproduction in any medium or format, as long as you give appropriate credit to the original author(s) and the source, provide a link to the Creative Commons license, and indicate if changes were made. The images or other third party material in this article are included in the article's Creative Commons license, unless indicated otherwise in a credit line to the material. If material is not included in the article's Creative Commons license and your intended use is not permitted by statutory regulation or exceeds the permitted use, you will need to obtain permission directly from the copyright holder. To view a copy of this license, visit http://creativecommons.org/ licenses/by/4.0/

(c) The Author(s) 2018 